\title{
Preface
}

\section{Randomized response techniques}

\author{
Sarjinder Singh \\ Editor in Chief
}

Collection of data on sensitive characteristics from human populations is not an easy task. For example sensitive questions: (a) Are you an Alawite? (b) Are you gay? (c) Have you ever molested a child? (d) Have you underreported your income on your tax return? (e) Do you smoke marijuana?, and (f) Have you ever cheated on an exam?, etc. These questions are not likely to be responded honestly by the respondents if they are asked using direct question survey methods. The randomized response technique was first introduced by Warner (1965) to deal with a problem of estimation of such sensitive characteristics in a finite population. This technique enables respondents to provide truthful information in anonymity on sensitive or highly personal questions without endangering their privacy.

To promote the pioneer idea of Warner (1965), we have devoted the present issue of the Journal Model Assisted Statistics and Applications on a special topic, "Randomized Response Techniques."

A number of prominent authors who work in the area of randomized response sampling have contributed towards to the special issue, and their details and contributions are explained in brief as follows:

Three famous authors: Lucio Barabesi, Department of Economics and Statistics, University of Siena, Italy; Giancarlo Diana, Department of Statistical Sciences, University of Padova, Italy, and Pier Francesco Perri, Department of Economics, Statistics and Finance, University of Calabria, Italy; have contributed a very decent article entitled, "Horvitz-Thompson Estimation with Randomized Response and Nonresponse." The three authors are from three different universities in Italy. It seems that randomized response technique is spreading across the whole Italy. These authors consider a situation where the randomized response theory is applied in a non-response set-up, and they adjusted the well known Horvitz-Thompson estimator for the scrambled responses.

An ingenious author Andreas Quatember, Department of Applied Statistics, Johannes Kepler University Linz, Austria (Europe) has contributed a decent article entitled, "A randomized response design for a polychotomous sensitive population and its application to opinion polls". He developed a new estimator which can be used in case of complex designs. He also discusses about the protection of respondents along with a technical issue of inadmissible estimates in randomized response technique. The contribution is unique, and if you read it you will not regret it. It shows that the use of idea of randomized response techniques is also well respected in European countries like Austria too not only Italy.

Four well known experts in the field of randomized response sampling: Ki-Hak Hong, Department of Computer Science, Dongshin University, South Korea; Gi-Sung Lee, Department of Children Welfare, Woosuk University, South Korea; Chang-Kyoon Son, Department of Statistics and Information Science, Dongguk University, South Korea; and Jong-Min Kim, Statistics Discipline, Division of Science and Mathematics, University of MinnesotaMorris, USA have contributed a very valuable article entitled, "An estimation of a sensitive attribute by two stage stratified randomized response model". These authors focus on the use of randomized response sampling using stratified random sampling which is a well known cost effective survey technique and works well when a population can be divided into homogeneous strata based on geographical or genetically related variables. The interesting part of the article is that this topic of randomized response techniques is so entertaining that it keeps scientist to go on thinking while they are working across different countries. It seems that three scientists from South Korea and one is from the USA are engaged with each other because of existence of randomized response technique. 
A young statistician Sally Abdelfatah (Ph.D. Scholar) and her advisor Reda Mazloum, Department of Statistics, Faculty of Economics and Political Science, Cairo University, Egypt, have taken a keen interest to work on randomized response techniques. Their new contribution in the present issue is, "Improved randomized response models using three decks of cards." Their recent contribution shows that a newly developed estimator has a lower bound of variance than the competitors in the literature.

Another energetic young statistician Tanveer A. Tarray (Ph.D. Scholar) and his untiring advisor Housila P. Singh, School of Studies in Statistics, Vikram University, India, have contributed two articles entitled, "An alternative to stratified Kim and Warde's randomized response model using optimal (Neyman) allocation" and "An improved mixed randomized response model." Their contribution in both articles is to consider and investigate the problem of estimation of sensitive characteristics in the setup of stratified random sampling for Neyman's allocation.

Three hard workers S.S. Sidhu, I.S. Grewal and M. Javed, Department of Mathematics, Statistics and Physics, Punjab Agricultural University, Ludhiana, India, also contributed an article entitled, "On estimation of totals of multi-sensitive-characteristics using forced quantitative randomized responses." Their claim is that an overview of the literature shows that when sensitive or incriminating topics are studied, the overall results of randomized response studies are more valid than the results of direct question designs. Thus they are proposing a set of alternative estimators for probability proportional to size with replacement corresponding to multi-character survey that elicit simultaneous information on many sensitive study variables.

Clearly a plea for the use of randomized response models in our daily life is self explanatory, and is being used in many countries across the world. By keeping such a plea of the use of randomized response devices in our daily life, we have devoted the present issue of the journal "Model Assisted Statistics and Applications" towards a new contribution in this particular field of specialization. These seven research papers which are included in this issue of the journal will be worth and valuable who wish to do future research in the area of randomized response sampling.

Recall that the pioneer model of randomized response technique was first introduced by Warner (1965). During 2015, we will be publishing another special issue entitled:

Golden Jubilee Year of the Warner's Randomized Response Model. 